\title{
Verstärkung im Treuhandnetz
}

Seit über 20 Jahren gehört die BMO Treuhand und Verwaltung AG zu den führenden Treuhandunternehmen im Kanton Schaffhausen. Für FMH-Mitglieder vertritt die BMO nun die Geschäftsstelle Neuhausen am Rheinfall.

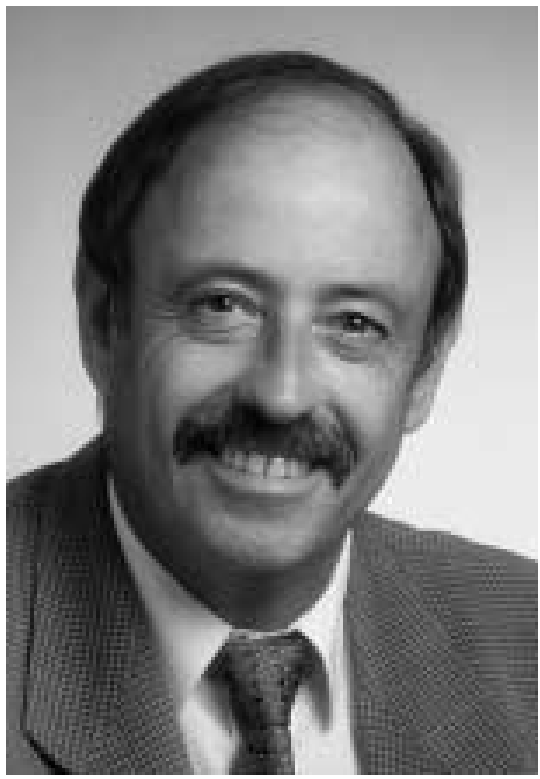

Peter Oechslin

lic. oec. publ, Partner, Geschäftsleitung BMO Leiter der Geschäftsstelle peter.oechslin@fmhtreuhand.ch

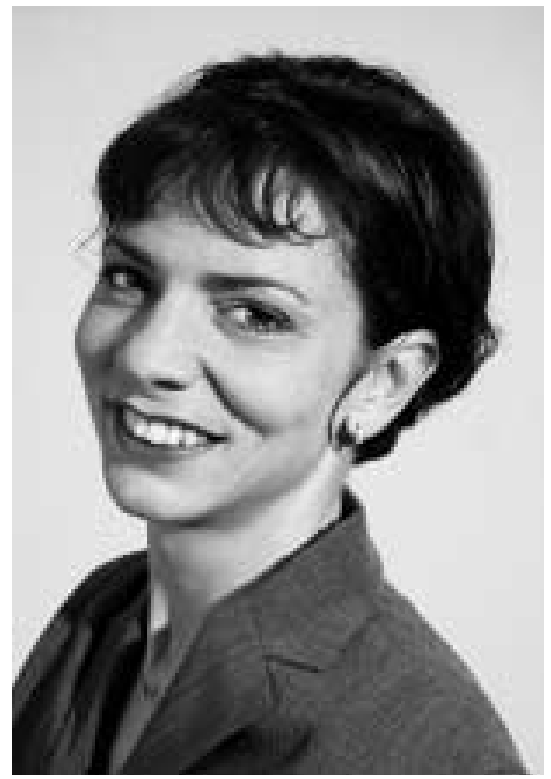

Katja Sigrist

Betriebsökonomin HWV

Stv. Leiterin der Geschäftsstelle katja.sigrist@fmhtreuhand.ch
FMH Services Treuhand Geschäftsstelle Neuhausen am Rheinfall

Rundbuckstrasse 6

8212 Neuhausen am Rheinfall

Tel. 0526755925

Fax 0526755920
Im Kanton Schaffhausen und grenzüberschreitend längst ein Begriff für Partnernähe und Qualität im Treuhandwesen, erweitert die BMO das FMH-Services-Treuhandstellennetz mit dem Ziel, dem Arzt in der Praxis ein breitmöglichstes Fachwissen in geographischer Nähe anzubieten.

Unternehmerisches Denken ist für die BMO auch in der Arztpraxis von ausschlaggebender Bedeutung. Nur eine optimale Betreuung im Back Office gewährt dem Mandanten die Möglichkeit, sich voll und ganz seiner Kernkompetenz zu widmen, um über kurz oder lang sein Unternehmen erfolgreich gestalten zu können. Die BMO unterstützt ihn dabei: Mit einem eingespielten Team von acht kompetenten Mitarbeitenden, die effiziente Lösungen für individuelle Problemstellungen finden - mit der gesamten Palette an treuhänderischen Dienstleistungen wie:

- Praxisgründungen und -auflösungen;

- Mithilfe beim Aufbau des Rechnungswesens;

- Führung von Finanz-, Betriebs- und Lohnbuchhaltungen;
- Steuerberatung;

- Rechtsberatung;

- Unternehmensberatung (z. B. Praxisübernahme/-übergabe).

Peter Oechslin und Katja Sigrist sind Ihre FMHServices-Ansprechpartner bei BMO. Sie stehen Ihnen für ein unverbindliches Gespräch gerne zu Diensten.

\section{Gemeinsamer Einkauf für Ärztinnen und Ärzte}

Die FMH Consulting arbeitet mit führenden Firmen für Arzt- und Spitalbedarf zusammen. Daneben arbeitet sie auch mit Partnern im Bereich Bürogeräte, Haushalt, Garten und Freizeit zusammen. Als Mitglied der FMH Services können Sie immer wieder von Sonderangeboten und Exklusivitäten profitieren.

Ferner möchten wir Ihnen helfen, Ihre monatlichen Zahlungen zu erleichtern, indem

- Sie direkt bei unseren Vertragslieferanten bestellen;

- die Vertragslieferanten der FMH Consulting Services die monatliche Abrechnung zusenden

- die FMH Consulting Services am 20. des Folgemonats diese Abrechnung bezahlt;

- Sie von der FMH Consulting Services jeweils am 15. des Monats eine Sammelrechnung erhalten.

\section{Unsere Vertragslieferanten sind die folgenden}

Aichele Medico AG, Basel

Apotheke zum Mörser, Zürich

Dr. F. Rappai, Schlieren

Dr. Grossmann AG, Birsfelden

Galexis AG, Schönbühl

IVF, Neuhausen

Kyocera Mita (Schweiz) AG, Volketswil

Medicare AG, Zürich

Mepha Pharma AG, Dornach

Mohren-Apotheke, Winterthur

Medpro Novamed AG, Flawil

Polymed MANERA Med. S.A., Froideville 
Polymed Medical Center, Glattbrugg

Raetus-Apotheke, Chur

Rufma AG, Bremgarten

Salzmann AG, St. Gallen

Spirig AG, Egerkingen

Spiromed, Ormalingen

Streuli \& Co. AG, Uznach

Uneltech Electronic AG, Schwerzenbach

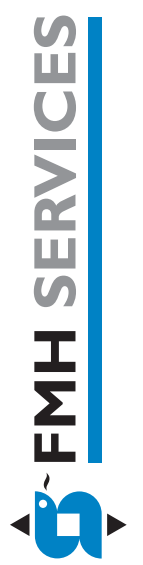

Unsere Treuhandspezialisten im deutschsprachigen Raum

Geschäftsstelle Muri b. Bern, Herr Peter Schneider, Tel. 0319518840

Geschäftsstelle Ostermundigen, Herr Harry Huwiler, Tel. 0319390139

Geschäftsstelle Olten,

Herr Peter Senn, Tel. 0622059035

Geschäftsstelle Basel,

Herr Linus Cavegn, Tel. 0613195121

Geschäftsstelle Lohn-Ammannsegg So,

Herr Rolf Lehmann, Tel. 0326775442

Geschäftsstelle Muri / AG,

Herr Roland Bütler, Tel. 0566640309

Geschäftsstelle Cham,

Herr Guido Schmid, Tel. 0417486290

Geschäftsstelle Sursee,

Herr Patrik Dahinden, Tel. 0419267045
Ihre Vorteile sind:

- Sie erhalten eine einmalige, monatliche Rechnung für alle Bezüge bei Vertragslieferanten während der letzten 4-6 Wochen.

- Ihr administrativer Aufwand beschränkt sich auf eine Zahlung.

- Sie haben weniger Bankspesen.

Möchten Sie in Zukunft bei einem unserer Partner einkaufen und über das Sammelrechungs-System abrechnen, dann genügt ein Anruf unter Tel. 04192500 77, FMH Consulting Services, Oberkirch.

Geschäftsstelle Stans,

Herr Marcel Helfenstein, Tel. 0416111821

Geschäftsstelle Zürich-Wiedikon,

Herr Christoph Lautenschlager, Tel. 014571575

\section{Geschäftsstelle Winterthur,}

Herr Urs Gross, Tel. 0522240241

Geschäftsstelle Weinfelden, Herr Adrian Hartmann, Tel. 0716228686

Geschäftsstelle Oberuzwil / SG, Herr Martin Brenner, Tel. 0719513066

Geschäftsstelle Speicher / St. Gallen, Herr Jürg Schmid, Tel. 0713442175

Geschäftsstelle Au / SG,

Herr Anibal Alghisi, Tel. 0717401787

Geschäftsstelle Chur,

p. Adr. Riedi Ruffner Theus AG, Tel. 0812584646

\section{www.FMHjob.ch}

Stellen- und Praxisvermittlung online

Für Fragen kontaktieren Sie unsere beiden Damen Frau Logovi und Frau Born von der Abteilung Stellenvermittlung in Bern unter Tel. 0313591212 oder E-Mail fmhstv@hin.ch.

\section{Service en ligne de placement et de remise de cabinets}

Si vous souhaitez obtenir de plus amples informations, n'hésitez pas à prendre contact avec Madame Logovi ou Madame Born de «l'Office de placement» à Berne au numéro de téléphone 0313591212 ou e-mail fmhstv@hin.ch.

\section{FMH Consulting Services}

Stellen- und Praxisvermittlung / Office de placement, Elfenstrasse 18, 3000 Bern 16, Tel./tél. 03135912 12, Fax/fax 03135911 12, E-Mail: fmhstv@hin.ch 